\title{
Coordination numbers in natural beach sand
}

\author{
Vanshan Wright ${ }^{1, *}$, Amy Ferrick ${ }^{2}$, Michael Manga ${ }^{2}$, and Nicholas Sitar $^{3}$ \\ ${ }^{1}$ Woods Hole Oceanographic Institution, Geology and Geophysics Department, Woods Hole, Massachusetts, USA, 02543 \\ ${ }^{2}$ University of California Berkeley, Department of Earth and Planetary Science, Berkeley, California, USA, 94720 \\ ${ }^{3}$ University of California Berkeley, Department of Civil and Environmental Engineering, Berkeley, California, USA, 94720
}

\begin{abstract}
Coordination number controls elastic moduli, seismic velocity, and force transmission in sands and is thus a critical factor controlling the resistance of sands to deformation. Previous studies quantified relationships between coordination number, porosity, grain size, sphericity, and effective stress in pluviated or modeled sands. Here, we determine if these relationships hold in naturally-deposited beach sands. We collect samples while preserving their microstructures and use x-ray computed microtomography images to characterize grain properties. Similar to pluviated and modeled sand studies, we find that average coordination numbers and porosities for freshly deposited natural sands are $8.1 \pm 2.8$ and $0.37 \pm 0.01$, respectively. The range and standard deviation in coordination numbers of the natural beach sands are, however, significantly higher than observed in pluviated and modeled sand studies. At the same effective stress and porosities, coordination number is linearly proportional to grain surface area except for the smallest and largest grains. Coordination number depends non-linearly on sphericity. We attribute the higher ranges and standard deviations of coordination numbers in the natural sands to its broader grain size distribution, and we propose that the largest grains limit grain rearrangement, which influences spatial distributions of coordination numbers in natural sands.
\end{abstract}

\section{Introduction}

Coordination number is the number of grains in contact. Coordination number affects the elastic moduli and seismic velocities of sands and is thus a critical parameter controlling how sand deforms. The coordination numbers of pluviated and modeled sands increase with decreasing porosity and increasing effective stresses [1-5] and decrease with decreasing sphericity [6]. An open question that we address is whether the same relationships exist in naturally-deposited sands. We answer this question by using x-ray computed microtomography to quantify relationships between coordination number, porosity, grain surface area, and sphericity in fresh and naturallydeposited sands at the Alameda County beach, Berkeley, California. Our results show that the average coordination numbers of these sands are roughly the same as predicted by existing pluviated and modeled sands studies but that naturally-deposited sands have higher variability in coordination numbers. Additional studies in natural systems over a broader range of effective stresses and physical properties (e.g., grain contact area, angularity, and sorting) are needed.

\section{Methods}

To determine which grain and pore properties affect coordination number, we first collected naturallydeposited beach sands with their microstructures preserved (section 2.1). We image the samples (section
2.2) to extract coordination number, sphericity, grain surface area, and porosity (section 2.3). We use correlation analyses to quantify relationships between these physical properties and coordination numbers.

\subsection{Sample Collection and Preparation}

We collected two partially saturated and undisturbed sand samples at Alameda County beach on July 11, 2020, at 10:15 am. The collection site was $\sim 7 \mathrm{~m}$ from the coastline at low tide. The collection depths were $6 \mathrm{~cm}$ and $11 \mathrm{~cm}$. To extract sand, we slowly inserted $2.0 \mathrm{~cm}$ long $1.2 \mathrm{~cm}$ diameter plastic straws into the sand. Once inserted, we excavate around the straw before extracting the straw. We tape the tops and bottoms of the sample and place the straws in a box surrounded by wet paper towel sheets. Storing the samples in this way limits evaporation, which could rearrange grains. Once at the home-lab (because COVID-19 is real), we wrap the sample in cheesecloth and encase the entire core in melted wax to seal and immobilize the samples further.

\subsection{Sample Imaging and Reconstruction}

We obtained $\mathrm{x}$-ray computed microtomography images on beamline 8.3.2 at the Advanced Light Source, Lawrence Berkeley National Lab. We used $30 \mathrm{keV}$ monochromatic x-rays, 200-millisecond exposure, and 1969 projections, which produced $\sim 500$ two-dimensional image slices per core. We imaged with a PCO edge

\footnotetext{
*Corresponding author: vwright@ whoi.edu

A video is available at https://doi.org/10.48448/bqjs-x193
} 
camera, a 1X Nikon lens, and a $50 \mathrm{~mm}$ LuAG scintillator while continuously rotating the sample through $180^{\circ}$. Isotropic pixel size is 6.45 microns. We reconstructed the images using the $\mathrm{Xi}$-cam software [7], including the center of rotation optimizations and removing rings and outliers.

\subsection{Image Segmentation and Analyses}

We segmented grains from pores to calculate grain and pore space properties. First, we binarize the images (grains versus pores) using the Trainable Weka Segmentation's machine learning algorithm in Fiji. This required training classifiers, which we created by applying a gaussian blur filter, performing an initial simple grayscale grain segmentation, and manually segmenting 5-10 grains on every $50^{\text {th }}$ image. The selected grains capture the ranges of grain sizes, shapes, sphericity, and grayscale values. We used the Distance Transform Watershed 3-D algorithm (distance map = Borgefors, dynamic $=2.0$, connectivity $=6$ ) to uniquely identify grains followed by a morphological closing filter (type = closing ball with a radius of 1 ) to re-establish contacts between touching grains. For quality control, we visually inspected segmentation results and estimated uncertainties by performing the segmentation process fifteen times, each time with new training classifiers. Once done, we used Software for Practical Analysis of Materials (SPAM) [8] to estimate coordination number, porosity, grain surface area, and sphericity from the segmented images. SPAM calculates sphericity as $36 \pi V^{2} A^{-3}$, where $V$ is volume and $A$ is surface area [8]. We report sphericity on a scale from 0 to 1 , where 1 is a perfect sphere.

\section{$3 \quad$ Results}

The key findings are that (1) the microstructures of the recovered sands are preserved, (2) the image processing method produces reliable estimates of coordination numbers, porosity, and grain surface area, and (3) the coordination number, grain surface area, and sphericity of the recovered sands co-vary.

\subsection{Uncertainty, Resolution, and Texture}

The collection method preserves the microstructures of naturally-deposited sands. The pore space and grains within the inner $9 \mathrm{~mm}$ of the naturally-deposited sands appear homogeneously distributed (Figure 1). Larger than average and irregularly positioned pores exist near the core walls (within $0.5 \mathrm{~mm}$ ), signaling possible grain disturbance (Figure 1). We thus analyze only the inner 9 $\mathrm{mm}$ diameter of the cores.

The segmentation process does not introduce significant uncertainties in the grain and pore properties. The analyzed region within each core contains $\sim 15,000$ grains. When we segment a subset of fifteen images with fifteen different classifiers, porosities vary by less than two percent and have a standard deviation of 0.016 . Using three separate classifiers to segment all 500 images in the same sample results in relatively small variations in the average coordination numbers [a percent change $(\mathrm{pc})$ of $6.9 \%$ and standard deviation (std) of 0.33 ], sphericity (pc $=1.3 \%$, std $=0.005)$, and grain surface area $(\mathrm{pc}=8.2 \%$, std $=0.008)$. Together, these results imply that the segmentation and image analysis methods produce high fidelity results.

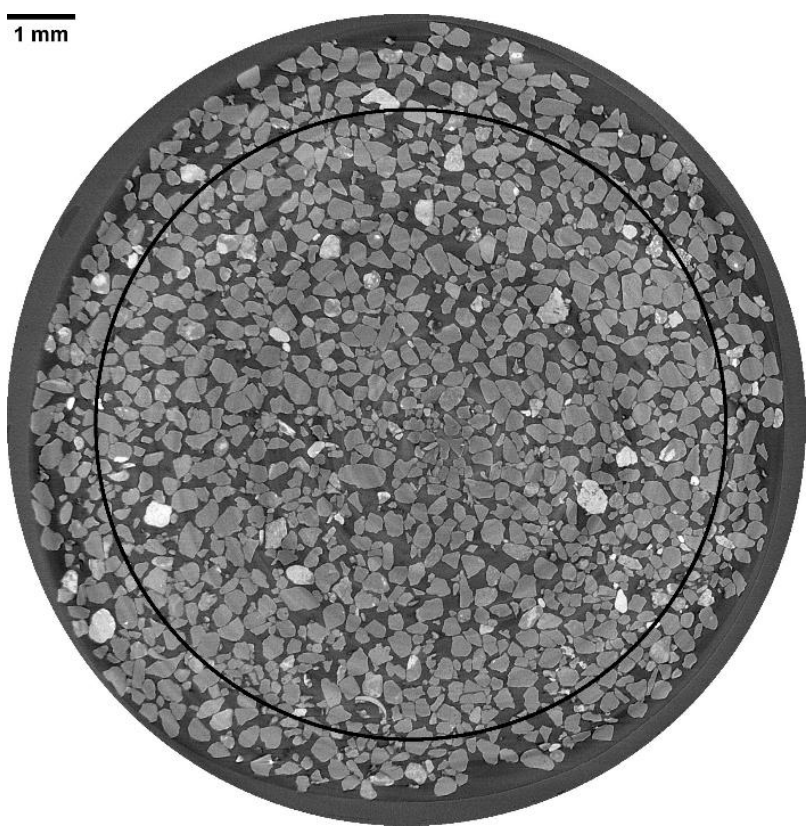

Figure 1. X-ray computed microtomography image showing a cross-section of one of the cores recovered at Alameda County beach. The black circle outlines the section of the image that we analyze. The outer grey ring is the plastic sampling tube.

\subsection{Grain and Pore Space Relationships}

The sands at Alameda County beach are mature (Figures 1-4). The respective porosities for the shallower and deeper samples are $0.37 \pm 0.01$ and $0.36 \pm 0.01$ (Figure 2). Grain surface area and sphericity (Figures 3-4) for the shallower and deeper samples are similar $\left(0.29 \mathrm{~mm}^{2} \pm\right.$ $0.01 \mathrm{~mm}^{2}$ and $0.24 \mathrm{~mm}^{2} \pm 0.01 \mathrm{~mm}^{2}$ for grains surface area versus $0.81 \pm 0.005$ and $0.82 \pm 0.005$ for sphericity, respectively). The average coordination number is lower in the shallower $(7.7 \pm 3.0)$ versus the deeper sample $(8.5$ \pm 3.4 ) (Figure $2 \mathrm{~b}$ ). Both samples' coordination numbers range from 2 to 20 (Figures 2-4).

Porosity, grain surface area, and sphericity influence coordination numbers. Coordination numbers of both samples increase with increasing grain surface area (Figure 3b). The coordination number of the samples increases with sphericity for coordination numbers less than five but decreases with increasing sphericity for coordination numbers that are six or greater (Figure $3 \mathrm{a}$ ). Coordination number moderately correlates $\left(r^{2}=0.56\right)$ with the combination of sphericity and grain surface area, assuming a linear relationship. The equation defining this relationship is $C=1.88 S+18.05 A+2.64$, where $C, S$, and $A$ are coordination number, sphericity, and surface area in $\mathrm{mm}^{2}$, respectively. Grain surface area affects coordination 

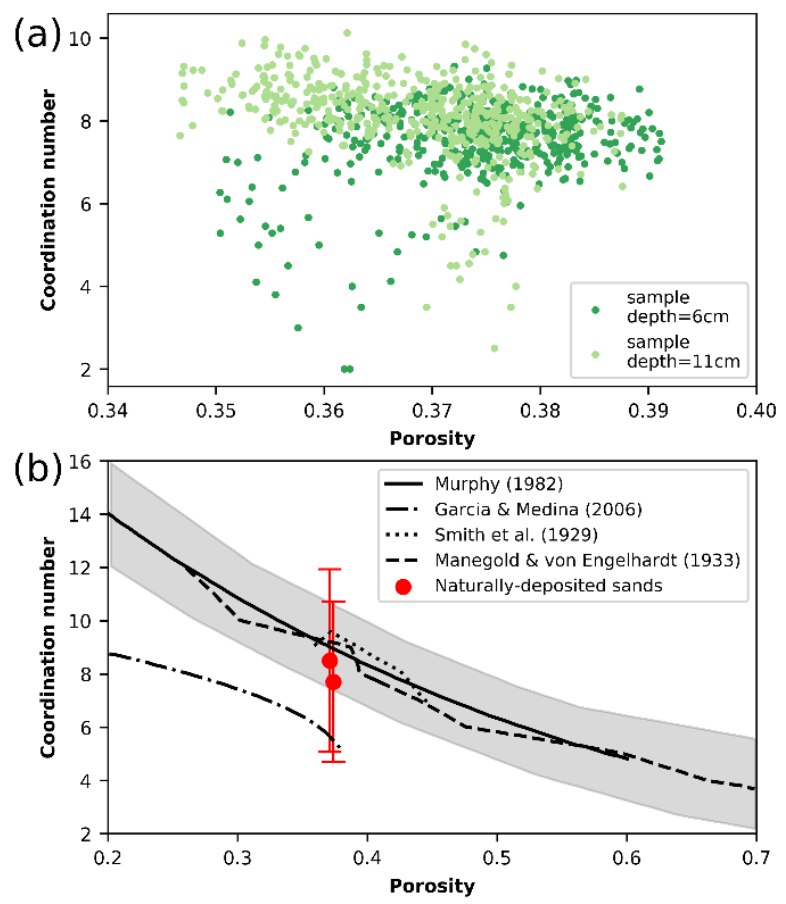

Figure 2. (a) Relationships between coordination number and porosity for recovered sands. We computed porosity and average coordination number on all 500 image slices within each core. (b) Comparison between coordination number for pluviated [1,3] and modeled [2,4] sands in the literature and our naturally-deposited sands. The gray shading is the standard deviation for the dataset produced by [2]. We modified the image from [5].
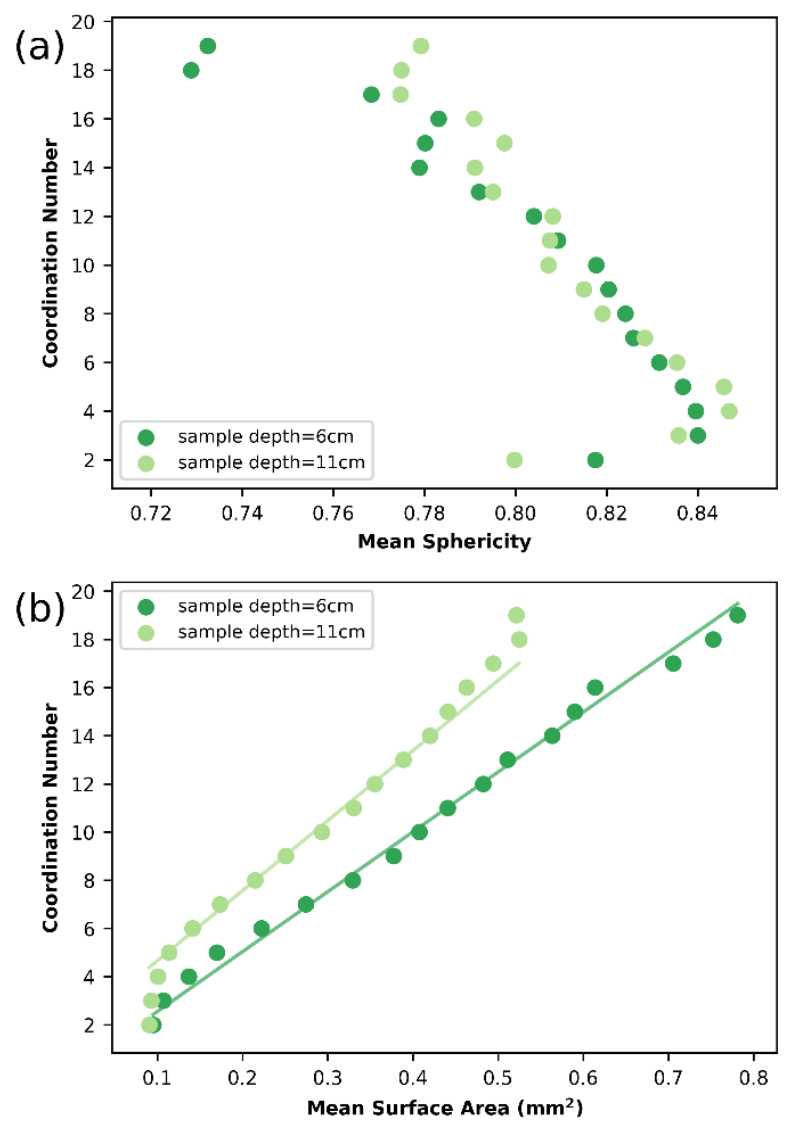

Figure 3. Relationship between coordination number and (a) sphericity and (b) grain surface area in recovered sands at Alameda County beach. number more substantially than sphericity (Figure 3-4). The differences in depth $(5 \mathrm{~cm})$ or porosities $(0.01)$ do not significantly influence the relationships between coordination number and sphericity. In contrast, coordination numbers rise faster with grain surface areas in the deeper sample (Figure 3b).
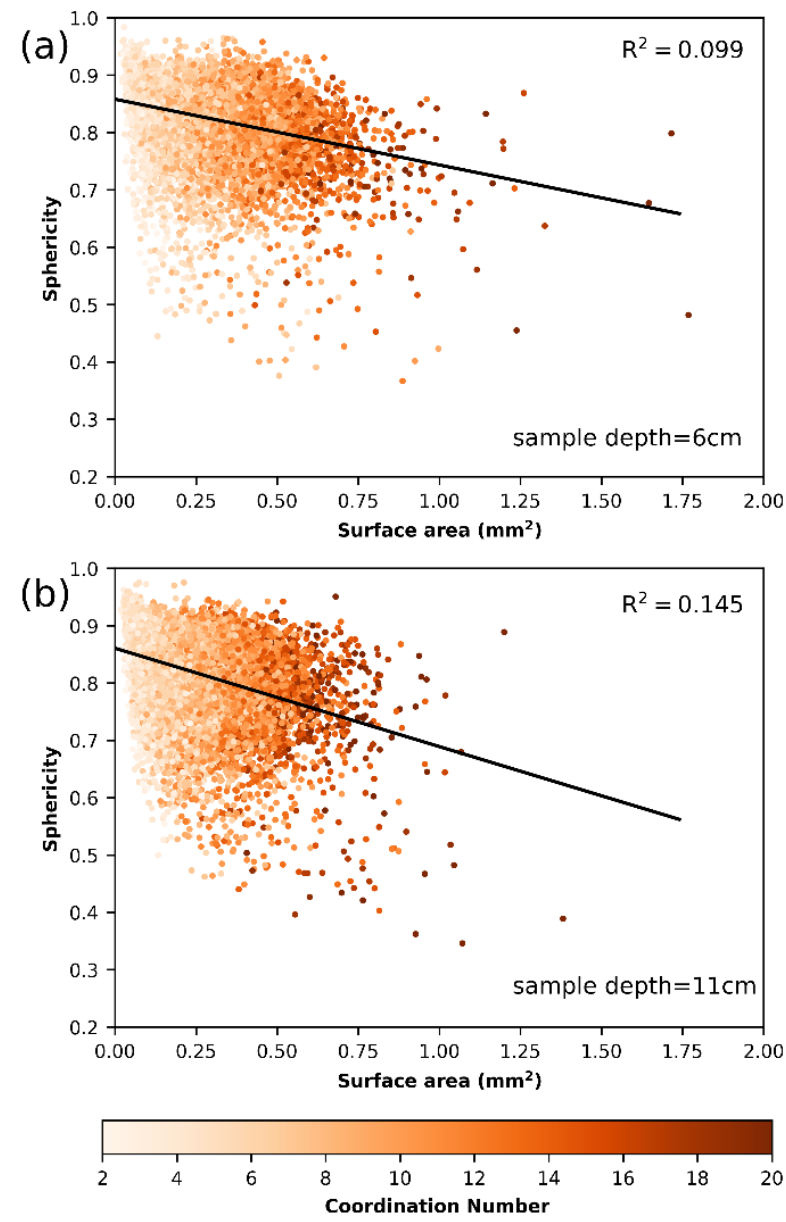

Figure 4. Relationship between coordination number, sphericity, and grain surface area for grains are analyzed in the (a) shallower and (b) deeper core. Covariance between sphericity and surface area is shown with a black line, assuming a linear relationship.

\section{Discussion}

Our results are generally consistent with previous modeled and pluviated loose sand studies; however, the relationships between coordination number, grain surface area, and sphericity (Figures 3-4) provide additional insights into grain packing processes at natural beaches. The porosities of the naturally-deposited sands are within range of the expected critical porosities of sands (i.e., $0.36-0.40$ ) [9]. Critical porosity is the porosity at which particles emerge from suspension, consolidate, behave as one unit, and are expected to have their lowest coordination number and highest porosities [9]. The average coordination numbers that we measure (i.e., $8.1 \pm$ $2.8)$ are expected for the porosities that we measure $(0.37$ \pm 0.01 ) (Figure 2b) [1-5]. Observations that the ranges and standard deviations of coordination numbers are greater in our samples (2-20 versus 4-12) [1-5] (Figures 2-4) are 
likely related to grain surface area, which exerts greater control on coordination numbers than sphericity (Figure 4). Since coordination number scales with surface area (Figure 3a, 4), the greater the range of surface area, the greater the range of coordination number.

We suggest that coordination number deviates from a linear scaling with grain surface area for the largest and smallest grains for two reasons. Small particles may be wedged between larger ones, making the surface of the small grains inaccessible to additional grain contacts. The largest grains also have fewer contacts than expected from the surface area scaling. We suggest that their large size blocks grains from rearranging around them, reducing the number of contacts. These interpretations highlight the role that sorting likely plays in controlling the coordination numbers and thus the elastic moduli of natural beach sands at or near critical porosity.

\section{Conclusion}

Coordination number is one of the most critical yet poorly constrained physical properties that affect the elastic moduli and seismic velocities of sands. Previous studies focus on determining what controls the coordination numbers in pluviated or model sands. In natural beach sands, we find that coordination number (1) averages 8.1 \pm 2.8 when porosities are $0.37 \pm 0.01$, which is consistent with existing pluviated and modeled sand studies, (2) increases with grain surface area except for the largest and smallest grains, (3) ranges from 2 to 20, and (4) nonlinearly depends on sphericity. Our future studies will use relationships between coordination numbers and a broader range of effective stresses and physical properties (e.g., grain contact area and angularity) to study what makes beach sands hard.

ALS-10575 enabled Advanced Light Source imaging. We thank Dr. Dula Parkinson for performing the imaging.

\section{References}

[1] W.O. Smith, P.D. Foote, P.G. Busand, Phys. Rev. 34, 1271-1274 (1929)

[2] E. Manegold, W. von Engelhardt, Kolloid Z. 63, 149-154 (1933)

[3] W.F. Murphy, Effects of microstructure and pore fluids on the acoustic properties of 316 granular sedimentary materials, Ph.D. dissertation, Stanford University (1982)

[4] X. Garcia, E.A. Medina, Geophysics 71, F13-F20 (2006)

[5] G. Mavko, T. Mukerji, J. Dvorkin, The Rock Physics Handbook, Cambridge University Press (2020)

[6] W. Fei, G.A. Narsilio, J. Geotech. Geoenviron. 146, 06020025 (2020)

[7] R.J. Pandolfi, D.B. Allan, E. Arenholz, L. BarrosoLuque, S.I. Campbell, T.A. Caswell, A. Blair, F. De Carlo, S. Fackler, A.P. Fournier, G. Freychet, J. Synchrotron Radiat. 25, 1261-1270 (2018)
[8] O. Stamati, E. Andò, E. Roubin, R. Cailletaud, M. Wiebicke, G. Pinzon, C. Couture, R. Hurley, R. Caulk, D. Caillerie, T. Matsushima, P. Bésuelle, F. Bertoni, T. Arnaud, A. Ortega Laborin, R. Rorato, Y. Sun, A. Tengattini, O. Okubadejo, J. Colliat, M. Saadatfar, F. Garcia, C. Papazoglou, I. Vego, S. Brisard, J. Dijkstra, G. Birmpilis, J. Open Source Softw. 5, 2286 (2020)

[9] A. Nur, G. Mavko, J. Dvorkin, D. Galmudi, Lead. Edge 17, 357-362 (1998) 